\title{
ON TRANSVERSE VIBRATIONS OF THIN, SHALLOW ELASTIC SHELLS*
}

\author{
BY \\ ERIC REISSNER \\ Massachusetts Institute of Terhnology
}

1. Introduction. This note is concerned with the form of the differential equations of the theory of shallow shells, as given by Marguerre [2], for the case of vibratory motion of the shell. In their general form these equations may be reduced to three simultaneous differential equations for three displacement components. It is our principal object here to show that for types of vibrations which we call transverse the equations of the theory-with negligible error-admit of an important simplification. This simplification consists in the omission of longitudinal inertia terms. It is possible with this omission to treat transverse vibrations by means of the same system of $t w o$ simultaneous equations for a stress function and for one displacement component which is known for the problems of statics of a shallow shell.

The advantages of the present treatment of the problem of transverse vibrations are illustrated by determining the frequencies of transverse vibrations of a shell with middle surface given by a second-degree equation if the shell is simply supported along a boundary with rectangular projection on the base plane.

We have previously used a special case of the results of this paper in the analysis of shallow arches [4]. Among other applications which are possible we mention the problem of the axisymmetrical vibrations of shallow spherical shells which has previously been considered by Federhofer [1] and the present writer [3], without taking advantage of the simplification which will be shown to be possible.

2. Basic differential equations. Marguerre's equations of the linear theory of shallow shells are here taken in the following form

$$
\begin{gathered}
\frac{\partial N_{x}}{\partial x}+\frac{\partial N_{x y}}{\partial y}=\rho h \frac{\partial^{2} u}{\partial t^{2}} \\
\frac{\partial N_{x y}}{\partial x}+\frac{\partial N_{\nu}}{\partial y}=\rho h \frac{\partial^{2} v}{\partial t^{2}} \\
\frac{\partial Q_{x}}{\partial x}+\frac{\partial Q_{\nu}}{\partial y}+\frac{\partial}{\partial x}\left[\frac{\partial z}{\partial x} N_{x}+\frac{\partial z}{\partial y} N_{x \nu}\right]+\frac{\partial}{\partial y}\left[\frac{\partial z}{\partial x} N_{x \nu}+\frac{\partial z}{\partial y} N_{\nu}\right]=\rho h \frac{\partial^{2} w}{\partial t^{2}} \\
\frac{\partial M_{x}}{\partial x}+\frac{\partial M_{x \nu}}{\partial y}-Q_{x}=0, \\
\frac{\partial M_{x \nu}}{\partial x}+\frac{\partial M_{\nu}}{\partial y}-Q_{\nu}=0 \\
M_{x}=-D\left(\frac{\partial^{2} w}{\partial x^{2}}+\nu \frac{\partial^{2} w}{\partial y^{2}}\right), \\
M_{\nu}=-D\left(\frac{\partial^{2} w}{\partial y^{2}}+\nu \frac{\partial^{2} w}{\partial x^{2}}\right),
\end{gathered}
$$

*Received August 5, 1954. A report on work supported by Office of Naval Research under Contract N5-ori-07834 with Massachusetts Institute of Technology. 


$$
\begin{gathered}
M_{x y}=-(1-\nu) D \frac{\partial^{2} w}{\partial x \partial y}, \\
\frac{\partial u}{\partial x}+\frac{\partial z}{\partial x} \frac{\partial w}{\partial x}=\frac{N_{x}-\nu N_{y}}{E h}, \\
\frac{\partial v}{\partial y}+\frac{\partial z}{\partial y} \frac{\partial w}{\partial y}=\frac{N_{y}-\nu N_{x}}{E h}, \\
\frac{\partial u}{\partial y}+\frac{\partial v}{\partial x}+\frac{\partial z}{\partial x} \frac{\partial w}{\partial y}+\frac{\partial z}{\partial y} \frac{\partial w}{\partial x}=\frac{N_{x \nu}}{G h} .
\end{gathered}
$$

In these equations $x, y$ are Cartesian coordinates, the equation of the middle surface of the shell being $z=z(x, y) ; u, v, w$ denote the displacement components along the coordinate axes, $t$ the time, $\rho$ the density, $h$ the shell thickness, $N$ the tangential stress resultants, $Q$ the transverse stress resultants, $M$ the stress couples, and $D=E h^{3} / 12\left(1-\nu^{2}\right)$ the bending stiffness.

Introduction of Eqs. (3) to (5) into (1) and (2) leads to three simultaneous equations for $u, v, w$. Restricting attention to cases for which $E$ and $h$ are constant these equations may be brought into the following form

$$
\begin{aligned}
& \frac{1-\nu}{2} \nabla^{2} u+\frac{1+\nu}{2} \frac{\partial}{\partial x}\left(\frac{\partial u}{\partial x}+\frac{\partial v}{\partial y}\right)+\frac{1-\nu}{2}\left(\frac{\partial z}{\partial x} \nabla^{2} w+\frac{\partial w}{\partial x} \nabla^{2} z\right) \\
& +\frac{1+\nu}{2} \frac{\partial}{\partial x}\left(\frac{\partial z}{\partial x} \frac{\partial w}{\partial x}+\frac{\partial z}{\partial y} \frac{\partial w}{\partial y}\right)=\frac{\rho}{E} \frac{\partial^{2} u}{\partial t^{2}} \\
& \frac{1-\nu}{2} \nabla^{2} v+\frac{1+\nu}{2} \frac{\partial}{\partial y}\left(\frac{\partial u}{\partial x}+\frac{\partial v}{\partial y}\right)+\frac{1-\nu}{2}\left(\frac{\partial z}{\partial y} \nabla^{2} w+\frac{\partial w}{\partial y} \nabla^{2} z\right) \\
& +\frac{1+\nu}{2} \frac{\partial}{\partial y}\left(\frac{\partial z}{\partial x} \frac{\partial w}{\partial x}+\frac{\partial z}{\partial y} \frac{\partial w}{\partial y}\right)=\frac{\rho}{E} \frac{\partial^{2} v}{\partial t^{2}} \\
& -D \nabla^{2} \nabla^{2} w+\frac{E h}{1-\nu^{2}}\left\{\frac{\partial^{2} z}{\partial x^{2}}\left[\frac{\partial u}{\partial x}+\frac{\partial z}{\partial x} \frac{\partial w}{\partial x}+\nu\left(\frac{\partial v}{\partial y}+\frac{\partial z}{\partial y} \frac{\partial w}{\partial y}\right)\right]\right. \\
& +\frac{\partial^{2} z}{\partial y^{2}}\left[\frac{\partial v}{\partial y}+\frac{\partial z}{\partial y} \frac{\partial w}{\partial y}+\nu\left(\frac{\partial u}{\partial x}+\frac{\partial z}{\partial x} \frac{\partial w}{\partial x}\right)\right] \\
& \left.+(1-\nu) \frac{\partial^{2} z}{\partial x \partial y}\left[\frac{\partial u}{\partial y}+\frac{\partial v}{\partial x}+\frac{\partial z}{\partial x} \frac{\partial w}{\partial y}+\frac{\partial z}{\partial y} \frac{\partial w}{\partial x}\right]\right\} \\
& =\rho h\left[\frac{\partial^{2} w}{\partial t^{2}}-\frac{\partial z}{\partial x} \frac{\partial^{2} u}{\partial t^{2}}-\frac{\partial z}{\partial y} \frac{\partial^{2} v}{\partial t^{2}}\right] \text {. }
\end{aligned}
$$

Equations equivalent to these have been used by Federhofer [1] and the writer [3] for the problem of axisymmetrical vibrations of shallow spherical shells. It is an indication of the difficulties associated with this system of equations that no successful attempt is known of an evaluation of the frequency determinant obtained from this theory for what is probably the simplest of these spherical-shell problems, the problem of the complete shell segment with clamped edge.

3. Simplified differential equations. It is well known that in problems of statics considerable practical advantage accrues if use is made of two simultaneous equations, for the displacement component $w$ and for an Airy stress function $F$ instead of three 
simultaneous equations for three displacements. In problems of dynamics this approach is prevented by the presence of the longitudinal inertia terms $u_{t t}$ and $v_{t t}$ in Eqs. (1). Suppose, however, that these terms are negligible for certain classes of dynamic problems. Then use of Airy's stress function becomes possible in the solution of these problems. Our principal aim in this note is concerned with just this point. We shall show that for classes of problems in which vibrations take place principally transversely we may neglect longitudinal inertia, at the same time when longitudinal straining may exert an important influence on the dynamic phenomenon.

Omitting $u_{t}$ and $v_{\iota t}$ in Eqs. (1) we set

$$
N_{x}=\frac{\partial^{2} F}{\partial y^{2}}, \quad N_{y}=\frac{\partial^{2} F}{\partial x^{2}}, \quad N_{x y}=-\frac{\partial^{2} F}{\partial x \partial y}
$$

and utilize a compatibility equation of the form

$$
\begin{aligned}
\frac{\partial^{2}}{\partial y^{2}}\left(\frac{\partial u}{\partial x}+\frac{\partial z}{\partial x} \frac{\partial w}{\partial x}\right)+\frac{\partial^{2}}{\partial x^{2}}\left(\frac{\partial v}{\partial y}+\frac{\partial z}{\partial y} \frac{\partial w}{\partial y}\right) & -\frac{\partial^{2}}{\partial x \partial y}\left(\frac{\partial u}{\partial y}+\frac{\partial v}{\partial x}+\frac{\partial z}{\partial x} \frac{\partial w}{\partial y}+\frac{\partial z}{\partial y} \frac{\partial w}{\partial x}\right) \\
& =2 \frac{\partial^{2} z}{\partial x \partial y} \frac{\partial^{2} w}{\partial x \partial y}-\frac{\partial^{2} z}{\partial x^{2}} \frac{\partial^{2} w}{\partial y^{2}}-\frac{\partial^{2} z}{\partial y^{2}} \frac{\partial^{2} w}{\partial x^{2}}
\end{aligned}
$$

Introduction of (5) and (7) into (8) gives the first of the two simultaneous equations for $F$ and $w$,

$$
\nabla^{2} \nabla^{2} F=E h\left\{2 \frac{\partial^{2} z}{\partial x \partial y} \frac{\partial^{2} w}{\partial x \partial y}-\frac{\partial^{2} z}{\partial x^{2}} \frac{\partial^{2} w}{\partial y^{2}}-\frac{\partial^{2} z}{\partial y^{2}} \frac{\partial^{2} w}{\partial x^{2}}\right\}
$$

Introduction of (3), (4) and (7) into (2) gives the second of the two simultaneous equations,

$$
D \nabla^{2} \nabla^{2} w+\rho h \frac{\partial^{2} w}{\partial t^{2}}=-2 \frac{\partial^{2} z}{\partial x \partial y} \frac{\partial^{2} F}{\partial x \partial y}+\frac{\partial^{2} z}{\partial x^{2}} \frac{\partial^{2} F}{\partial y^{2}}+\frac{\partial^{2} z}{\partial y^{2}} \frac{\partial^{2} F}{\partial x^{2}} .
$$

4. Justification of simplified differential equations. In order to establish conditions under which neglect of longitudinal inertia is justified we undertake an order-of-magnitude analysis of the displacement differential equations (6). We introduce dimensionless variables

$$
\xi=\frac{x}{l}, \quad \eta=\frac{y}{l}, \quad \tau=\omega t
$$

and

$$
w=W f, \quad u=U g, \quad v=V k, \quad(10 \mathrm{a}, \mathrm{b}, \mathrm{c})
$$

such that the functions $f, g, k$ and their derivatives with respect to $\xi, \eta, \tau$ are at most of order of magnitude unity. We further set

$$
\begin{aligned}
& z_{x}=\frac{H}{L} \zeta_{x}, \quad z_{y}=\frac{H}{L} \zeta_{y}, \\
& z_{x x}=\frac{H}{L^{2}} \zeta_{x x}, \quad z_{y y}=\frac{H}{L^{2}} \zeta_{y y}, \quad z_{x y}=\frac{H}{L^{2}} \zeta_{x y}, \quad(12 \mathrm{a}, \mathrm{b}, \mathrm{c})
\end{aligned}
$$

where $\zeta_{x}, \zeta_{y}, \zeta_{x x}, \zeta_{y y}, \zeta_{x y}$ are at most of order of magnitude unity and where $(H / L)^{2}$ is negligibly small compared with unity, in order that the theory of shallow shells be applicable. 
We now consider terms of all representative types in Eq. (6). From (6a),

$$
\frac{U}{l^{2}} g_{\xi \xi}+\frac{H}{L} \zeta_{x} \frac{W}{l^{2}} f_{\xi \xi}+\frac{H}{L^{2}} \zeta_{x x} \frac{W}{l} f_{\xi}+\cdots=\frac{\rho \omega^{2}}{E} U g_{\tau \tau} .
$$

Equation (6b), being analogous in structure, need not be considered separately. Equation (6c) becomes

$$
\begin{gathered}
\frac{E h^{3}}{12\left(1-\nu^{2}\right)} \frac{W}{l^{4}}\left[-f_{\xi \xi \xi \xi}+\cdots\right]+\frac{E h}{1-\nu^{2}} \frac{H}{L^{2}} \zeta_{x x}\left\{\left[\frac{U}{l} g_{\xi}+\frac{H}{L} \zeta_{x} \frac{W}{l} f_{\xi}\right]+\cdots\right\}+\cdots \\
=\rho h \omega^{2}\left[W f_{\tau \tau}-\frac{H}{L} \zeta_{x} U g_{r \tau}-\cdots\right] .
\end{gathered}
$$

We are free to dispose of four quantities in (13), $U, W, l$ and $\omega$. Appropriate disposition leads to the results which are desired.

Since we expect that longitudinal straining will exert an influence on transverse vibrations we must see to it that in (13a) terms involving both $f$ and $g$ remain significant. This is accomplished by setting either

$$
U=\frac{H}{L} W
$$

or, if $l / L \gg 1$,

$$
U=\frac{H}{L} \frac{l}{L} W
$$

This transforms (13a) into

$$
g_{\xi \xi}+\zeta_{x} f_{\xi \xi}+\frac{l}{L} \zeta_{x x} f_{\xi}+\cdots=\frac{\rho \omega^{2} l^{2}}{E} g_{r r}
$$

or

$$
g_{\xi \xi}+\frac{L}{l} \zeta_{x} f_{\xi \xi}+\zeta_{x x} f_{\xi}+\cdots=\frac{\rho \omega^{2} l^{2}}{E} g_{r r} .
$$

Since terms in (15) can be at most of order unity we conclude that (14a) and (15a) are applicable as long as

$$
\left.\frac{l}{L}=O(1), \quad \text { (including } \ll 1\right),
$$

while (14b) and (15b) are applicable as long as

$$
\left.\frac{L}{l}=O(1), \quad \text { (including } \ll 1\right) .
$$

Case (16a) means that representative vibration wave lengths $l$ are at most of the order of magnitude of representative shell shape wave lengths $L$. Case (16b) includes the case of vibration-wave lengths which are large compared to shell shape wave lengths. Case (16a) is of importance in problems such as that of the shallow spherical shell. Case (16b) concerns problems such as the problem of vibrations of corrugated plates. In either case it may happen that the coefficient on the right of $(15), \rho \omega^{2} l^{2} / E$, is small compared to unity. If this does happen then longitudinal inertia will be negligible. 
To obtain information on this point we consider next (13b) with either (14a) or (14b). From (13b) and (14a)

$$
\begin{aligned}
& \frac{1}{12\left(1-\nu^{2}\right)} \frac{h^{3}}{l^{4}}\left[-f_{\xi \xi \xi \xi}+\cdots\right]+\frac{1}{1-\nu^{2}} \frac{H^{2} h}{L^{3} l} \zeta_{x x}\left\{\left[g_{\xi}+\zeta_{x} f_{\xi}\right]+\cdots\right\} \\
& +\cdots=\frac{\rho h \omega^{2}}{E}\left[f_{r \tau}-\frac{H^{2}}{L^{2}} \zeta_{x} g_{r \tau}+\cdots\right] .
\end{aligned}
$$

From (13b) and (14b),

$$
\begin{array}{r}
\frac{1}{12\left(1-\nu^{2}\right)} \frac{h^{3}}{l^{4}}\left[-f_{\xi \xi \xi \xi}+\cdots\right]+\frac{1}{1-\nu^{2}} \frac{H^{2} h}{L^{4}} \zeta_{x x}\left\{\left[g_{\xi}+\zeta_{x} \frac{L}{l} f_{\xi}\right]+\cdots\right\} \\
+\cdots=\frac{\rho h \omega^{2}}{E}\left[f_{\tau \tau}-\frac{H^{2}}{L^{2}} \frac{l}{L} \zeta_{x} g_{\tau \tau}-\cdots\right] .
\end{array}
$$

Since transverse inertia is to be important, it is necessary to match the coefficient of $f_{r r}$ with at least one of the coefficients on the left side of (17). We consider first (17a). We set

$$
\frac{1}{12\left(1-\nu^{2}\right)} \frac{h^{2}}{l^{4}}=\frac{\rho \omega^{2}}{E}
$$

or

$$
\frac{1}{1-\nu^{2}} \frac{H^{2}}{L^{3} l}=\frac{\rho \omega^{2}}{E}
$$

With this, taking account of the fact that $(H / L)^{2} \ll 1$,

$$
\left[-f_{\xi \xi \xi \xi}+\cdots\right]+12 \frac{H^{2}}{h^{2}} \frac{l^{3}}{L^{3}} \zeta_{x x}\left\{\left[g_{\xi}+\zeta_{x} f_{\xi}\right]+\cdots\right\}+\cdots=f_{r r}
$$

or

$$
\frac{1}{12} \frac{L^{3}}{l^{3}} \frac{h^{2}}{H^{2}}\left[-f_{k \xi \xi \xi}+\cdots\right]+\zeta_{x x}\left\{\left[g_{\xi}+\zeta_{x} f_{\xi}\right]+\cdots\right\}+\cdots=f_{r} \quad \quad(19 \mathrm{a}, \text { ii })
$$

Equation $(19 \mathrm{a}, \mathrm{i})$ is applicable as long as

$$
12 \frac{H^{2}}{h^{2}} \frac{l^{3}}{L^{3}}=O(1) \quad(\text { or } \ll 1)
$$

while $(19 \mathrm{a}, \mathrm{ii})$ is applicable when

$$
\frac{1}{12} \frac{L^{3}}{l^{3}} \frac{h^{2}}{H^{2}}=O(1) \quad(\text { or } \ll 1),
$$

the two regions of parameter values complementing each other.

We now introduce $\rho \omega^{2} / E$ from (18a) into (15a). The coefficient on the right of (15a) becomes

$$
\frac{1}{12\left(1-\nu^{2}\right)} \frac{h^{2}}{l^{2}}
$$


and

$$
\frac{1}{1-\nu^{2}} \frac{H^{2}}{L^{2}} \frac{l}{L}
$$

respectively. We may assume that we have, besides $H^{2} / L^{2} \ll 1$ also $h^{2} / l^{2} \ll 1$. In fact, it is only if this assumption is made that the effect of transverse shear deformation can be neglected, as we have done at the outset. Since $l / L=O(1)$, we have then that both the above longitudinal inertia coefficients are small compared with unity. Accordingly, as long as representative wave length $l$ and frequency $\omega$ are related as in $(18 \mathrm{a}, \mathrm{i})$ or $(18 \mathrm{a}$, ii) and as long as $l / L=O(1)$ we have that the effect of longitudinal inertia in the theory of shallow shells is negligible compared with the effect of transverse inertia. We remark that further simplifications of the basic differential equations are possible if

$$
\frac{l}{L} \ll 1 \quad \text { and (or) } \quad 12 \frac{H^{2}}{h^{2}} \frac{l^{3}}{L^{3}} \ll 1 \quad \text { or } \quad \frac{1}{12} \frac{L^{3}}{l^{3}} \frac{h^{2}}{H^{2}} \ll 1,
$$

provided, in the third case, that the boundary conditions are such that bending need not occur.

We now consider case (14b), (16b) and (17b) for which $l / L$ may be large compared with unity. We set in (17b)

or

$$
\frac{1}{12\left(1-\nu^{2}\right)} \frac{h^{2}}{l^{4}}=\frac{\rho \omega^{2}}{E}
$$

$$
\frac{1}{1-\nu^{2}} \frac{H^{2}}{L^{4}}=\frac{\rho \omega^{2}}{E} \text {. }
$$

This gives

$$
\begin{aligned}
{\left[-f_{\xi \xi \xi \xi}+\cdots\right]+12 \frac{H^{2}}{h^{2}} \frac{l^{4}}{L^{4}} \zeta_{x x}\left\{\left[g_{\xi}+\zeta_{x} \frac{L}{l} f \xi\right]\right.} & +\cdots\} \\
& +\cdots=f_{r r}-\frac{H^{2}}{L^{2}} \frac{l}{L} g_{r r}-\cdots
\end{aligned}
$$

or

$$
\begin{aligned}
\frac{1}{12} \frac{h^{2}}{H^{2}} \frac{L^{4}}{l^{4}}\left[-f_{\xi \xi \xi \xi}+\cdots\right]+\zeta_{x x}\left\{\left[g_{\xi}+\zeta_{x} \frac{L}{l} f_{\xi}\right]\right. & +\cdots\} \\
& +\cdots=f_{r r}-\frac{H^{2}}{L^{2}} \frac{l}{L} \zeta_{x} g_{r r}-\cdots,
\end{aligned}
$$

while (15b) becomes

$$
g_{\xi \xi}+\zeta_{x x} f_{\xi}+\frac{L}{l} \zeta_{x} f_{\xi \xi}+\cdots=\left\{\begin{array}{l}
\frac{1}{12\left(1-\nu^{2}\right)} \frac{h^{2}}{l^{2}} g_{\tau r} \\
\frac{1}{1-\nu^{2}} \frac{H^{2} l^{2}}{L^{4}} g_{\tau r} .
\end{array}\right.
$$

Equations $(19 \mathrm{~b}, \mathrm{i})$ and $(21, \mathrm{i})$ can be further simplified. Since

$$
\frac{H^{2} l^{4}}{h^{2} L^{4}}=\frac{H^{2}}{L^{2}} \frac{l^{2}}{h^{2}} \frac{l^{2}}{L^{2}}=O(1),
$$


in the range $L / l=O(1)$ and $l / h \gg 1$, it follows that $(H / L)^{2}(l / L) \ll 1$ and the second term on the right of $(19 \mathrm{~b}, \mathrm{i})$ is negligible. Furthermore, the longitudinal inertia term in (21, i) is also negligible. Accordingly, the simplified equations (I) and (II) are applicable even when $l / L \gg 1$, provided only that $(18 \mathrm{~b}, \mathrm{i})$ holds, that is provided that plate bending action contributes significantly.

It is not possible to simplify Eqs. (19b, ii) and (21, ii) further since we are not allowed to conclude that the inertia terms $(H / L)^{2}(l / L) g_{\tau \tau}$ and $(H / L)^{2}(l / L)^{2} g_{r \tau}$ are always negligibly small. Accordingly, when

$$
\frac{l}{L} \gg 1 \quad \text { and } \quad \frac{1}{12} \frac{h^{2}}{H^{2}} \frac{L^{4}}{l^{4}}=O(1),
$$

the simplified equations (I) and (II) may not be applicable.

5. Vibrations of paraboloidal shell with rectangular boundary. We consider as a simple illustration of the use of the foregoing results a shell with middle surface equation

$$
z=c_{0}+\frac{1}{2} c_{1} x^{2}+\frac{1}{2} c_{2} y^{2}
$$

simply supported along edges $x= \pm a$ and $y= \pm b$. We further assume that the supporting structures are rigid in their own planes but non-resistant perpendicular to their own planes.

The boundary conditions for $w$ are

$$
\begin{array}{lll}
x= \pm a ; & w=0, & \frac{\partial^{2} w}{\partial x^{2}}=0 \\
y= \pm b ; & w=0, & \frac{\partial^{2} w}{\partial y^{2}}=0 .
\end{array}
$$

The boundary conditions for $F$ can be shown to be

$$
\begin{array}{lll}
x= \pm a ; & \frac{\partial^{2} F}{\partial y^{2}}=0, & \frac{\partial^{2} F}{\partial x^{2}}=0, \\
y= \pm b ; & \frac{\partial^{2} F}{\partial x^{2}}=0, & \frac{\partial^{2} F}{\partial y^{2}}=0 .
\end{array}
$$

The differential equations (I) and (II) reduce to the following form

$$
\begin{gathered}
\nabla^{2} \nabla^{2} F=-E h\left\{c_{1} \frac{\partial^{2} w}{\partial y^{2}}+c_{2} \frac{\partial^{2} w}{\partial x^{2}}\right\}, \\
D \nabla^{2} \nabla^{2} w=-\rho h \frac{\partial^{2} w}{\partial t^{2}}+c_{1} \frac{\partial^{2} F}{\partial y^{2}}+c_{2} \frac{\partial^{2} F}{\partial x^{2}} .
\end{gathered}
$$

The form of these equations and of the boundary conditions (23) and (24) allows the following modes of vibration.

$$
\begin{aligned}
& w=A e^{i \omega t}\left\{\begin{array}{l}
\cos \alpha_{2 n+1} x \cos \beta_{2 n+1} y \\
\sin \alpha_{2 n} x \sin \beta_{2 n} y
\end{array}\right. \\
& F=B e^{i \omega t}\left\{\begin{array}{l}
\cos \alpha_{2 n+1} x \cos \beta_{2 n+1} y \\
\sin \alpha_{2 n} x \sin \beta_{2 n} y
\end{array}\right.
\end{aligned}
$$


where

$$
\alpha_{m}=\frac{m \pi}{2 a}, \quad \beta_{m}=\frac{m \pi}{2 b} .
$$

Introduction of (26) into (25) gives

$$
\begin{gathered}
E h\left(c_{1} \beta_{q}^{2}+c_{2} \alpha_{p}^{2}\right) A_{p q}-\left(\alpha_{p}^{2}+\beta_{q}^{2}\right)^{2} B_{p q}=0, \\
{\left[D\left(\alpha_{p}^{2}+\beta_{a}^{2}\right)^{2}-\rho h \omega_{p q}^{2}\right] A_{p q}+\left(c_{1} \beta_{a}^{2}+c_{2} \alpha_{p}^{2}\right) B_{p q}=0,}
\end{gathered}
$$

where $p$ and $q$ may be any integers greater than or equal to unity.

Vanishing of the determinant of (28) leads to a frequency equation of the form

$$
\omega_{p q}^{2}=\frac{D}{\rho h}\left(\alpha_{p}^{2}+\beta_{a}^{2}\right)^{2}+\frac{E}{\rho}\left(\frac{c_{2} \alpha_{p}^{2}+c_{1} \beta_{a}^{2}}{\alpha_{p}^{2}+\beta_{q}^{2}}\right)^{2} .
$$

Spherical cap with quadratic base. For a more detailed discussion of the frequency equation (29) we choose a shell for which

$$
c_{0}=H, \quad c_{1}=\frac{-H}{a^{2}}, \quad c_{2}=\frac{-H}{a^{2}}, \quad a=b .
$$

Introduction of (30) into (29) transforms (29) into the following form

$$
\omega_{p Q}^{2}=\frac{\pi^{4}\left(p^{2}+q^{2}\right)^{2}}{192\left(1-\nu^{2}\right)} \frac{E}{\rho} \frac{h^{2}}{a^{4}}\left[1+\frac{192\left(1-\nu^{2}\right)}{\pi^{4}\left(p^{2}+q^{2}\right)^{2}} \frac{H^{2}}{h^{2}}\right] .
$$

The frequency $\omega_{p Q}$ is smallest when $p=q=1$. The value of $\omega_{11}$ is given by,

$$
\omega_{11}^{2}=\frac{\pi^{4}}{48\left(1-\nu^{2}\right)} \frac{E}{\rho} \frac{h^{2}}{a^{4}}\left[1+\frac{48\left(1-\nu^{2}\right)}{\pi^{4}} \frac{H^{2}}{h^{2}}\right] .
$$

It is seen that the shell-curvature correction term is relatively largest for the lowest mode and becomes significant as soon as $H / h$ is about unity.

The frequency equation (31) may be compared with the relations (18a) which were involved in the justification of the use of (I) and (II). We have here both $L$ and $l$ of order of magnitude $a$. Accordingly, $\omega$ as given by (31) is included in the range of admissible values which is determined by $(18 \mathrm{a}, \mathrm{i})$ and $(18 \mathrm{a}, \mathrm{ii})$.

The following further observation may be made. As $H / h$ increases the order of magnitude of the frequencies of transverse vibrations increases from $(E / \rho)^{1 / 2}\left(h / a^{2}\right)$ to $(E / \rho)^{1 / 2}\left(H / a^{2}\right)$. On the other hand the order of magnitude of the frequencies of longitudinal vibrations of flat plates is $(E / \rho)^{1 / 2}(1 / a)$. Since $H / a \ll 1$ for shallow shells, we see that, while shell curvature increases frequencies of transverse vibrations in relation to the corresponding flat plate frequencies, these shell frequencies remain of a lower order than longitudinal flat-plate vibration frequencies. It would seem that this fact is the physical reason why the transverse vibrations of shallow shells take place with negligible amount of longitudinal inertia.

\section{REFERENCES}

1. K. Federhofer, Zur Berechnung der Eigenschwingungen der Kugelschale, Sitzungber. Akad. d. Wiss. Wien 146, 57-69 (1937)

2. K. Marguerre, Zur T'heorie der gekrünmten Platte grosser Formänderung, Proc. Vth Intern. Congr. Appl. Mech., 93-101 (1938)

3. E. Reissner, On vibrations of shallow spherical shells, J. Appl. Phys. 17, 1038-1042 (1946)

4. E. Reissner, Note on the problem of vibrations of slightly curved bars, J. Appl. Mech. 21, 195-196 (1954) 